\title{
Collective Thomson scattering measurements of fast-ion transport due to sawtooth crashes in ASDEX Upgrade
}

\author{
Rasmussen, Jesper; Nielsen, Stefan Kragh; Pedersen, Morten Stejner; Galdon-Quiroga, J.; Garcia- \\ Munoz, M.; Geiger, B.; Jacobsen, Asger Schou; Jaulmes, F.; Korsholm, Søren Bang; Lazanyi, N. \\ Total number of authors: \\ 16
}

Published in:

Nuclear Fusion

Link to article, DOI:

10.1088/0029-5515/56/11/112014

Publication date:

2016

Document Version

Peer reviewed version

Link back to DTU Orbit

Citation (APA):

Rasmussen, J., Nielsen, S. K., Pedersen, M. S., Galdon-Quiroga, J., Garcia-Munoz, M., Geiger, B., Jacobsen, A. S., Jaulmes, F., Korsholm, S. B., Lazanyi, N., Leipold, F., Ryter, F., Salewski, M., Schubert, M., Stober, J., \& Wagner, D. (2016). Collective Thomson scattering measurements of fast-ion transport due to sawtooth crashes in ASDEX Upgrade. Nuclear Fusion, 56(11), 112014. https://doi.org/10.1088/0029-5515/56/11/112014

\section{General rights}

Copyright and moral rights for the publications made accessible in the public portal are retained by the authors and/or other copyright owners and it is a condition of accessing publications that users recognise and abide by the legal requirements associated with these rights.

- Users may download and print one copy of any publication from the public portal for the purpose of private study or research.

- You may not further distribute the material or use it for any profit-making activity or commercial gain

- You may freely distribute the URL identifying the publication in the public portal 


\section{EUROfusion}

EUROFUSION WPMST1-PR(16)14817

J. Rasmussen et al.

\section{Collective Thomson Scattering Measurements of Fast-Ion Transport Due to Sawtooth Crashes in ASDEX Upgrade}

Preprint of Paper to be submitted for publication in

Nuclear Fusion 
"This document is intended for publication in the open literature. It is made available on the clear understanding that it may not be further circulated and extracts or references may not be published prior to publication of the original when applicable, or without the consent of the Publications Officer, EUROfusion Programme Management Unit, Culham Science Centre, Abingdon, Oxon, OX14 3DB, UK or e-mail Publications.Officer@euro-fusion.org".

"Enquiries about Copyright and reproduction should be addressed to the Publications Officer, EUROfusion Programme Management Unit, Culham Science Centre, Abingdon, Oxon, OX14 3DB, UK or e-mail Publications.Officer@euro-fusion.org".

The contents of this preprint and all other EUROfusion Preprints, Reports and Conference Papers are available to view online free at http://www.euro-fusionscipub.org. This site has full search facilities and e-mail alert options. In the JET specific papers the diagrams contained within the PDFs on this site are hyperlinked. 


\title{
Collective Thomson scattering measurements of fast-ion transport due to sawtooth crashes in ASDEX Upgrade
}

\author{
J Rasmussen ${ }^{1}$, S K Nielsen ${ }^{1}$, M Stejner ${ }^{1}$, B Geiger $^{2}$, \\ A S Jacobsen ${ }^{1}$, F Jaulmes ${ }^{3}$, S B Korsholm ${ }^{1}$, F Leipold ${ }^{1}$, \\ F Ryter ${ }^{2}$, M Salewski ${ }^{1}$, M Schubert ${ }^{2}$, J Stober ${ }^{2}$, D Wagner $^{2}$, \\ the ASDEX Upgrade Team ${ }^{2}$, the EUROFusion MST1 Team ${ }^{4}$ \\ 1 Technical University of Denmark, Department of Physics, Fysikvej, building 309, \\ DK-2800 Kgs. Lyngby, Denmark \\ ${ }^{2}$ Max-Planck-Institut für Plasmaphysik, Boltzmannstr. 2, D-85748 Garching, \\ Germany \\ ${ }^{3}$ FOM Institute DIFFER, 3430 BE Nieuwegein, The Netherlands \\ ${ }^{4}$ http://www.euro-fusionscipub.org/mst1 \\ E-mail: jeras@fysik.dtu.dk
}

\begin{abstract}
Sawtooth instabilities can modify heating and current-drive profiles and potentially increase fast-ion losses. Understanding how sawteeth redistribute fast ions as a function of sawtooth parameters and of fast-ion energy and pitch is hence a subject of particular interest for future fusion devices. Here we present the first collective Thomson scattering (CTS) measurements of sawtooth-induced redistribution of fast ions at ASDEX Upgrade. These also represent the first localized fast-ion measurements on the high-field side of this device. The results indicate fast-ion losses in the phasespace measurement volume of about $50 \%$ across sawtooth crashes, in good agreement with values predicted with the Kadomtsev sawtooth model implemented in TRANSP and with the sawtooth model in the EBdyna_go code. In contrast to the case of sawteeth, we observe no fast-ion redistribution in the presence of fishbone modes. We highlight how CTS measurements can discriminate between different sawtooth models, in particular when aided by multi-diagnostic velocity-space tomography, and briefly discuss our results in light of existing measurements from other fast-ion diagnostics.
\end{abstract}

\section{Introduction}

Fast ions are used for heating and current drive in present-day fusion devices, and fast fusion-born $\alpha$-particles will play a key role in heating the plasma in future burningplasma machines. However, these ions can also interact strongly with a range of corelocalized MHD modes, causing increased fast-ion losses and heating of the first wall [1]. Understanding the behaviour and transport of fast ions in the presence of these modes is hence important for assessing the fusion performance and stable operating regimes of future devices. One mode which can interact strongly with fast ions in the plasma core is 
the sawtooth instability [2], which redistributes heat, momentum, and particles radially outwards, including large populations of fast ions. A key challenge is to understand how this redistribution depends on fast-ion energy and pitch as well as on plasma parameters and the sawtooth crash amplitude or period.

Collective Thomson Scattering (CTS) is well suited for studies of the mechanisms underlying fast-ion redistribution by sawteeth, given its flexible measurement geometry which allows measurements in specific regions of real space and fast-ion velocity space. CTS is based on injecting an electromagnetic probe beam into the plasma and receiving part of the radiation scattered off (mainly ion-driven) fluctuations in the electron distribution. Its versatility makes CTS useful for measuring a range of parameters of both thermal and fast-ion populations [3]. CTS is also well suited for reactorrelevant conditions, and a CTS system is currently being developed for fast $\alpha$-particle measurements in ITER (e.g. [4]). In sawtooth experiments at TEXTOR, CTS was used to show that passing fast ions are more susceptible to strong sawtooth-induced redistribution than trapped fast ions [5], a result that has been subsequently confirmed using fast-ion $\mathrm{D}_{\alpha}$ spectroscopy (FIDA) on other devices such as DIII-D [6] and ASDEX Upgrade (AUG) [7]. FIDA measurements [8,9] have also indicated that the sawtooth redistribution of fast ions at AUG is generally well described by the widely used Kadomtsev sawtooth model [10].

Along with FIDA, CTS is the only diagnostic at AUG which can provide corelocalized fast-ion measurements in broad regions of fast-ion velocity space. It is thus of great interest to complement and extend earlier fast-ion results on AUG by CTS measurements, also since these are sensitive to different regions in fast-ion velocity space than, e.g., FIDA. Recently, the installation of a dedicated CTS receiver for background monitoring has helped significantly to improve the acquisition and analysis of CTS data at AUG [11]. CTS measurements of thermal and energetic ions in MHDquiescent discharges now show good agreement with results from other diagnostics and with neo-classical theory [12]. Building on these improvements, we here present the first CTS results on fast-ion interactions with sawteeth at AUG (representing the first fast-ion measurements localized on the AUG high-field side), and we compare the results to predictions of the Kadomtsev model and the recently developed full-orbit code EBdyna_go [13].

\section{Measurements and analysis}

The results presented here are based on AUG discharge 30382, with a plasma current $I_{p}=1.0 \mathrm{MA}$, toroidal magnetic field $B_{t}=-2.65 \mathrm{~T}$, and a relatively low central lineintegrated density $N_{e} \lesssim 3 \times 10^{19} \mathrm{~m}^{-2}$ during our experiments. Neutral beam injection (NBI) of fast deuterium ions with the co-current on-axis NBI source Q3 (D injection energy of $60 \mathrm{keV}$ ) was active from $t=2.0-3.0 \mathrm{~s}$, with no other auxiliary heating applied. As illustrated in the time traces shown in figure 1, regular sawteeth appeared during this phase, indicated by variations in central soft X-ray data and in core ion temperature 

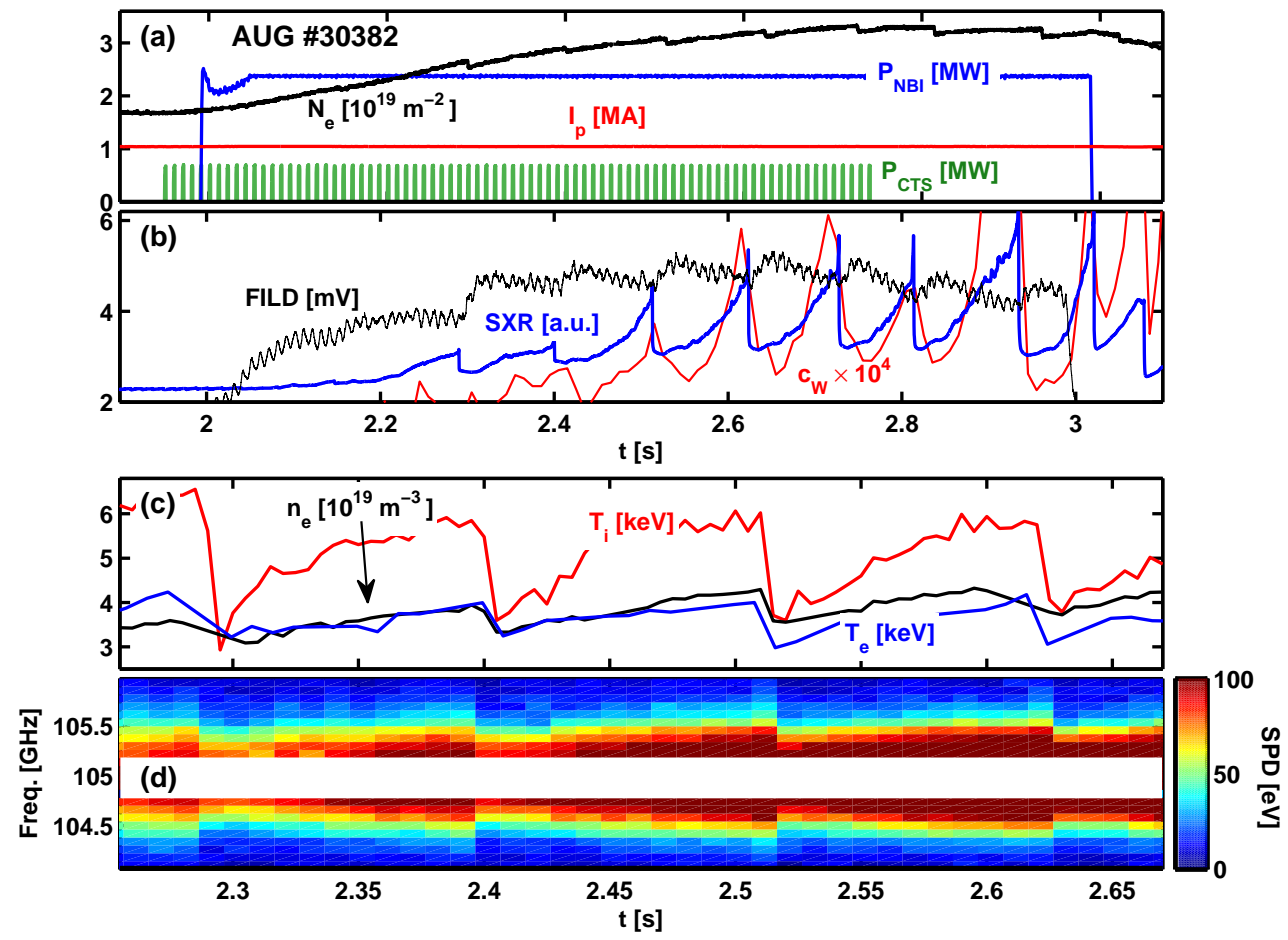

Figure 1. Timetraces of AUG discharge 30382. (a) Central line-integrated electron density, NBI power, plasma current, and CTS probe gyrotron power. (b) Central soft $\mathrm{X}$-ray emission, tungsten concentration from $E>2 \mathrm{keV}$ line radiation, and calibrated signal from the fast-ion loss detectors (FILD; [14]). (c) Local ion temperature from charge exchange recombination spectroscopy and local electron density from integrated data analysis, both interpolated to the flux coordinate of the CTS volume, along with core electron temperature from Thomson scattering. (d) CTS spectrogram centered on the probe gyrotron frequency, showing spectral power densities outside the stopbands of the central notch filters.

and electron temperature and density. The sawtooth crash amplitude in temperature and density is seen to remain fairly constant, whereas that of the X-ray signal rises due to an increase in the tungsten line radiation.

CTS data were acquired concurrently with most of the NBI phase, using the dualreceiver setup discussed in [11]. The $105 \mathrm{GHz}$ probing gyrotron $(P \simeq 600 \mathrm{~kW}$, weakly absorbed O-mode polarization) was modulated in a duty cycle with $2 \mathrm{~ms}$ on-periods and $8 \mathrm{~ms}$ off-periods to enable subtraction of the background which is dominated by electron cyclotron emission (ECE). Accurate background subtraction is critical for the present work, since CTS fast-ion measurements are sensitive to rapid variations in the ECE background such as those occurring during sawtooth events. The CTS measurement volume, defined by the overlap of the gyrotron probe beam with the receiver view, was placed slightly on the high-field side at $(R, z)=(1.62,0.06) \mathrm{m}$. CTS measurements are sensitive to the projection of the fast-ion velocity distribution function onto the plasma fluctuation vector $\mathbf{k}^{\delta}$, where $\mathbf{k}^{\delta}$ is defined by the orientation 


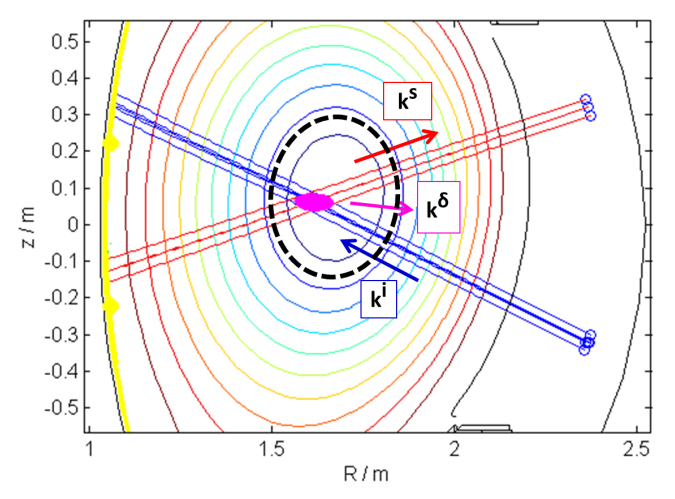

Figure 2. Poloidal view of the CTS scattering geometry. The incoming gyrotron probe beam (blue; wave vector $\mathbf{k}^{i}$ ) scatters off plasma fluctuations along $\mathbf{k}^{\delta}$ in the CTS measurement volume (magenta), to produce radiation $\left(\mathrm{red} ; \mathbf{k}^{s}=\mathbf{k}^{i}+\mathbf{k}^{\delta}\right.$ ) detectable by the CTS receiver. The dashed ellipse outlines $\rho_{p}=0.4$, the approximate location of the sawtooth inversion radius.

of the probe beam and receiver view, as illustrated in figure 2. Here $\mathbf{k}^{\delta}$ had an angle of $\phi=\angle\left(\mathbf{k}^{\delta}, \mathbf{B}\right)=101^{\circ}$ relative to the local magnetic field. The measurement location corresponds to a normalized poloidal flux of $\rho_{p} \simeq 0.15$ and is well inside the sawtooth inversion radius at $\rho_{p} \approx 0.4$ as estimated from soft $\mathrm{X}$-ray measurements. Indeed, the resulting CTS spectrogram shown in figure 1(d), which represents the time evolution of the background-subtracted CTS spectrum, clearly shows how the spectra respond to variations in plasma properties across sawtooth crashes. The spectra are seen to broaden during the recovery phases between crashes, in part due to the build-up and subsequent ejection of fast ions from the measurement volume and in part due to the sawtoothing behaviour of bulk plasma parameters such as $T_{i}$ and $n_{e}$.

In order to interpret the observed spectral variations across sawtooth crashes, the data are analyzed using a fully electromagnetic forward model of the scattering [15]. This model employs measurements of thermal bulk plasma parameters from other diagnostics wherever available, including electron density and temperature from integrated data analysis [16] and ion temperature and toroidal rotation velocity from charge exchange recombination spectroscopy on boron, all interpolated to the flux coordinate of the measurement volume. The scattering geometry and location as estimated from raytracing are also included.

Fast ions are included in the forward model using the distribution function in the scattering volume predicted with TRANSP/NUBEAM [17], run with the Kadomtsev model for the fast-ion redistribution at sawtooth crashes. In the TRANSP/Kadomtsev implementation, fast ions are treated in the guiding center approximation and remain bound to the evolving magnetic field lines, which undergo full reconnection during a sawtooth crash. We also considered the post-crash distribution function predicted by the full-orbit EBdyna_go code [13] as comparison. In addition to assuming full Kadomtsev reconnection, this code computes the evolution of the electromagnetic fields during a 
sawtooth collapse based on [18] and evaluates the full particle orbits subject to these evolving fields (allowing for particle detachment from the evolving flux surfaces). To ensure a meaningful comparison with TRANSP, the EBdyna_go simulations used the pre-crash fast-ion distribution functions predicted with TRANSP as initial conditions.

From fits to the measured spectra using the above scattering model, the projection of the fast-ion velocity distribution function $f(\mathbf{v})$ onto $\mathbf{k}^{\delta}$ can also be inferred. The result is the $1 \mathrm{D}$ (fast) ion velocity distribution $g(u)=\int f(\mathbf{v}) \delta\left(\mathbf{v} \cdot \mathbf{k}^{\delta} / k^{\delta}-u\right) \mathrm{d} \mathbf{v}$ in the scattering volume as a function of projected velocity $u$. Here $\delta(\ldots)$ is the Dirac $\delta$-function. The fitting is done within a Bayesian framework, with no functional form assumed for $g(u)$, and with uncertainties on all priors (e.g., values of bulk plasma parameters, impurity concentrations, and CTS scattering angles) taken into account. Further details on the forward modelling and the fitting procedure can be found in, e.g., [12, 19].

\section{Results}

The CTS measurement period shown in figure 1 covers four clear sawtooth crashes, whereas measurements later in the discharge (not shown) probed much less prominent crashes. Here we focus mainly on the two crashes taking place at $t=2.29 \mathrm{~s}$ and $2.51 \mathrm{~s}$. These occur for slightly different electron densities and impurity concentrations (figure 1), and they display different sawtooth crash durations as discussed below. This allows us to explore the impact of sawteeth under varying plasma conditions. In addition, these crashes are preceded by relatively weak precursor or fishbone activity compared to the two other crashes. Since such activity can also contribute to anomalous fast-ion transport, the selected two crashes thus offer a relatively clean view of the fastion redistribution by the sawtooth crashes themselves.

In figure 3, we show measured CTS spectra before and after the two sawtooth crashes. The CTS data have here been averaged over two gyrotron on-periods to improve the signal-to-noise ratio, giving an effective total integration time of $20 \mathrm{~ms}$ for CTS and

background measurements. This is short compared with the sawtooth period of more than $100 \mathrm{~ms}$ (figure 1). The results are compared to our forward model generated as described above, with fast ions included from TRANSP. Overall, the measurements show good agreement with the forward model. In particular, the measured spectra become clearly narrower across sawtooth crashes in agreement with the model, also at frequency shifts of $\Delta f \gtrsim 0.7 \mathrm{GHz}$ from the probe gyrotron frequency, where the forward model suggests that the measurements are strongly dominated by fast ions.

At these frequency shifts, the spectral power density is seen to decrease by about $50 \%$ across the two crashes. As mentioned, this decrease results from ejection of fast ions from the measurement volume as well as from sawtoothing behavior of bulk plasma parameters such as the electron density. To search for a similar effect in the fitted 1D fast-ion velocity distribution function, we compare in figure 4 the inferred $g(u)$ to the corresponding TRANSP predictions. Following the approach in [12], TRANSP model uncertainties of $25 \%$ have been included in the figure, based on the typical uncertainties 

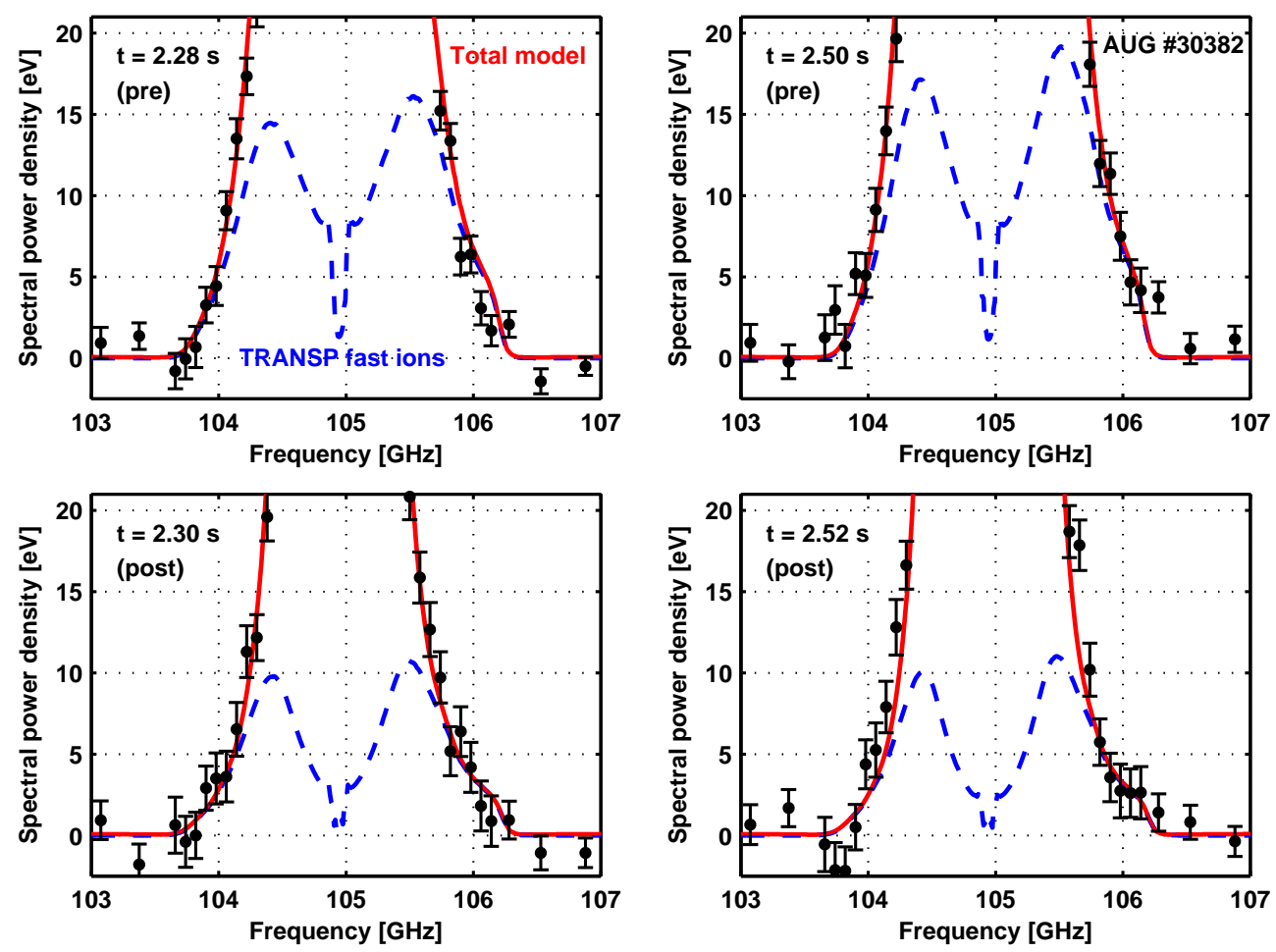

Figure 3. Pre- and post-crash CTS spectra for AUG \#30382 compared to synthetic spectra from the corresponding forward model (solid red) and its fast-ion contribution from TRANSP (dashed blue) for the sawtooth crashes at (left) $t=2.29 \mathrm{~s}$ and (right) $2.51 \mathrm{~s}$. Uncertainties on measured data represent the standard error of the mean at a given frequency within the relevant probe gyrotron pulses.

in the kinetic profiles $\left(N_{e}, T_{e}, T_{i}\right)$ within the sawtooth inversion radius at the relevant times. Within these computational and experimental uncertainties, there is generally good agreement between TRANSP and the CTS results.

The CTS measurements clearly suggest a lower fast-ion content following the crash at $t=2.51 \mathrm{~s}$, with indications, although less significant, of a fast-ion reduction also after the $t=2.29 \mathrm{~s}$ crash. To quantify this, we evaluate 1D partial fast-ion densities by integrating the inferred $g(u)$ over the velocity interval $1.5 \times 10^{6} \mathrm{~m} / \mathrm{s}<|u|<3.5 \times 10^{6} \mathrm{~m} / \mathrm{s}$, for which both pre- and post-crash distributions are well defined by our fits. The results indicate a decrease in the fast-ion density in the observed velocity space of $40 \pm 24 \%$ and $60 \pm 22 \%$ at $t=2.29 \mathrm{~s}$ and $2.51 \mathrm{~s}$, respectively. The quoted uncertainties here represent the propagated errors on the partial fast-ion densities, based on Monte Carlo realizations of $g(u)$ where the value of $g$ at each velocity node $u$ was repeatedly drawn from a Gaussian distribution with width and mean equal to the experimentally inferred value and its uncertainty, respectively. The results imply statistically significant fast-ion redistribution at levels consistent with the corresponding values of $44 \%$ and $56 \%$ predicted with TRANSP. Hence, our measurements provide the first evidence of sawtooth redistribution of fast ions measured by CTS at AUG and suggest redistribution 

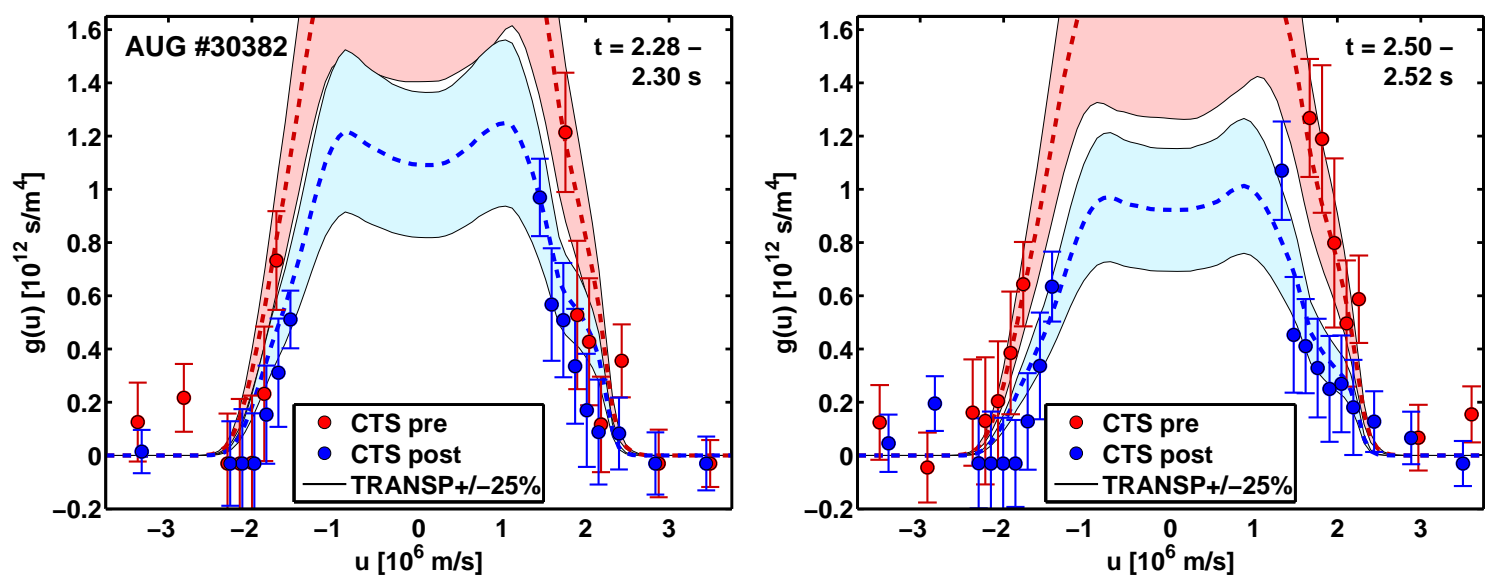

Figure 4. Fast-ion distribution functions from CTS data as a function of projected velocity before and after the sawtooth crashes at (left) $t=2.29 \mathrm{~s}$ and (right) $2.51 \mathrm{~s}$. The corresponding TRANSP predictions and their uncertainties are shown as dashed lines and filled regions, respectively.

levels which are well matched by the Kadomtsev model in TRANSP within the uncertainties. If considering positive and negative projected velocities separately, we observe a stronger redistribution for $u>0$ than for $u<0$; for the crash at $t=2.51 \mathrm{~s}$, the fast-ion reduction is $67 \pm 23 \%$ for $1.5 \times 10^{6} \mathrm{~m} / \mathrm{s}<u<3.5 \times 10^{6} \mathrm{~m} / \mathrm{s}$, but only $38 \pm 35 \%$ for the corresponding negative velocity interval. At $t=2.29 \mathrm{~s}$, the redistribution level is, in fact, significantly different from zero only at $u>0$. We will return to the origin of this asymmetry in projected velocity space in section 4 .

For comparison to TRANSP, we also consider the more detailed fast-ion treatment incorporated in the EBdyna_go code, run here with sawtooth crash durations of $100 \mu \mathrm{s}$ (at $t=2.29 \mathrm{~s}$ ) and $125 \mu \mathrm{s}(t=2.51 \mathrm{~s}$ ), as suggested by soft X-ray data (see [20] for details). To guide the discussion, it is instructive to first consider the simulated distribution function $f$ from TRANSP and EBdyna_go around the crash times, as well as highlight some differences in the two modelling approaches. In figure 5 we plot $f(E, p)$ in the measurement volume in energy-pitch coordinates, with pitch $p=v_{\|} / v$ (where $v_{\|}$is the ion velocity component anti-parallel to the magnetic field, at AUG defined positive in the co-current direction). Recall that, by construction, the pre-crash distribution functions of the two codes are identical, so for EBdyna_go we only show the post-crash predictions. Also note that the EBdyna_go runs discussed here employ a coarser velocity-space grid than our TRANSP simulations, due to the computational loads associated with the full-orbit calculations. Finally, we point out that the TRANSP output has been averaged over $2 \mathrm{~ms}$ (corresponding to the CTS gyrotron on-period) and accounts for the continuous fuelling and slowing down of NBI particles during this time interval. In contrast, the EBdyna_go simulation includes no source or collision terms and has been run at a high time resolution of a few $\mu \mathrm{s}$, but for a shorter total duration of $0.5 \mathrm{~ms}$. These differences can at least partly explain the significantly higher post- 

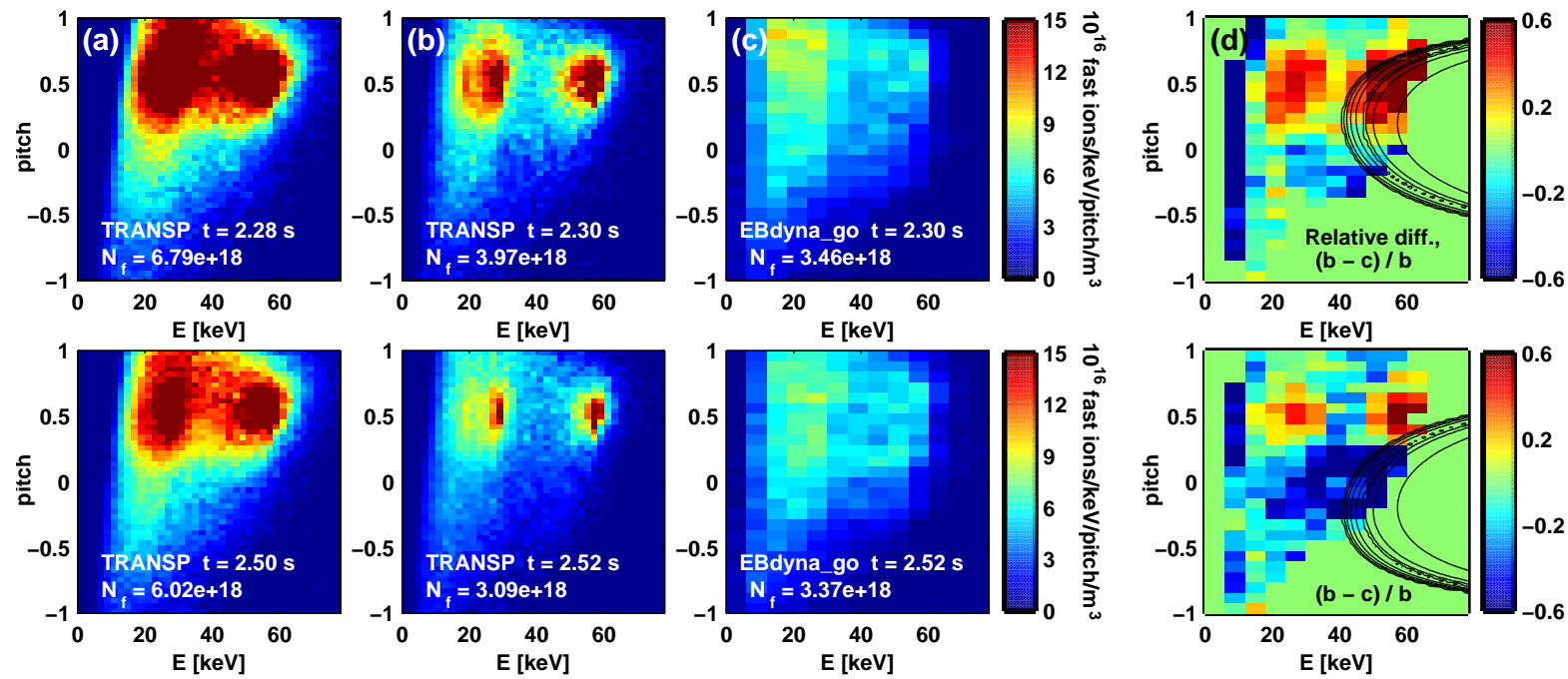

Figure 5. Fast-ion velocity distribution functions $f(E, p)$ in the CTS volume for AUG \#30382, as predicted by (a) TRANSP at pre-crash, (b) TRANSP post-crash, and (c) EBdyna_go post-crash, for (top) the crash at $t=2.29 \mathrm{~s}$ and (bottom) $t=2.51 \mathrm{~s}$. Labels give the local fast-ion density, $N_{\mathrm{f}}=\iint f(E, p) \mathrm{d} E \mathrm{~d} p\left[\mathrm{~m}^{-3}\right]$. Panel $(\mathrm{d})$ shows the relative post-crash difference between the models, $\left(f_{\text {TRANSP }}-\right.$ $\left.f_{\text {EBdyna_go }}\right) / f_{\text {TRANSP }}$, with $f_{\text {TRANSP }}$ resampled to match the coarser parameter grid employed by the EBdyna_go simulation. Contours show CTS weight functions for (top) $u=+2 \times 10^{6} \mathrm{~m} / \mathrm{s}$ and (bottom) $u=-2 \times 10^{6} \mathrm{~m} / \mathrm{s}$.

crash density of ions close to the NBI injection peaks seen in the TRANSP simulation compared to EBdyna_go. While keeping this in mind, the EBdyna_go model is seen to predict a stronger overall redistribution than TRANSP at $t=2.29 \mathrm{~s}$ when integrated over fast-ion energy and pitch. However, a weaker impact on fast ions than implied by TRANSP is predicted at $t=2.51 \mathrm{~s}$, in particular for ions with pitches close to zero, see figure $5(\mathrm{~d})$. This latter point highlights the fact that, at fixed plasma location, the two codes predict different redistribution patterns in $(E, p)$ space.

Figure 5 also shows CTS weight functions $W[4]$, which illustrate the sensitivity of a CTS measurement $S$ at a given projected velocity $u$ and projection angle $\phi=\angle\left(\mathbf{k}^{\delta}, \mathbf{B}\right)$ to different regions in fast-ion velocity space, such that $S=\iint W(E, p) f(E, p) \mathrm{d} E \mathrm{~d} p$ $[4,21]$. In the adopted scattering geometry $\left(\phi=101^{\circ}\right)$, the CTS signal is seen to contain a contribution from ions around the full-energy $(E=60 \mathrm{keV})$ injection peak for a projected velocity of $u=+2 \times 10^{6} \mathrm{~m} / \mathrm{s}$ (but not at $-2 \times 10^{6} \mathrm{~m} / \mathrm{s}$ ). Weight functions for other projected velocities are similar in appearance but slightly broader and shifted toward lower energies for lower $|u|$. Even though CTS is sensitive to both passing and trapped ions in the present setup, the central high-field side location of the CTS volume implies that virtually all fast ions in the CTS volume are passing, according to TRANSP. Hence, these particular measurements cannot test the prediction of EBdyna_go that trapped and passing ions are affected differently above a certain critical energy [18] (estimated to be $E_{\text {crit }} \approx 35-45 \mathrm{keV}$ for the discharge and sawtooth parameters used 

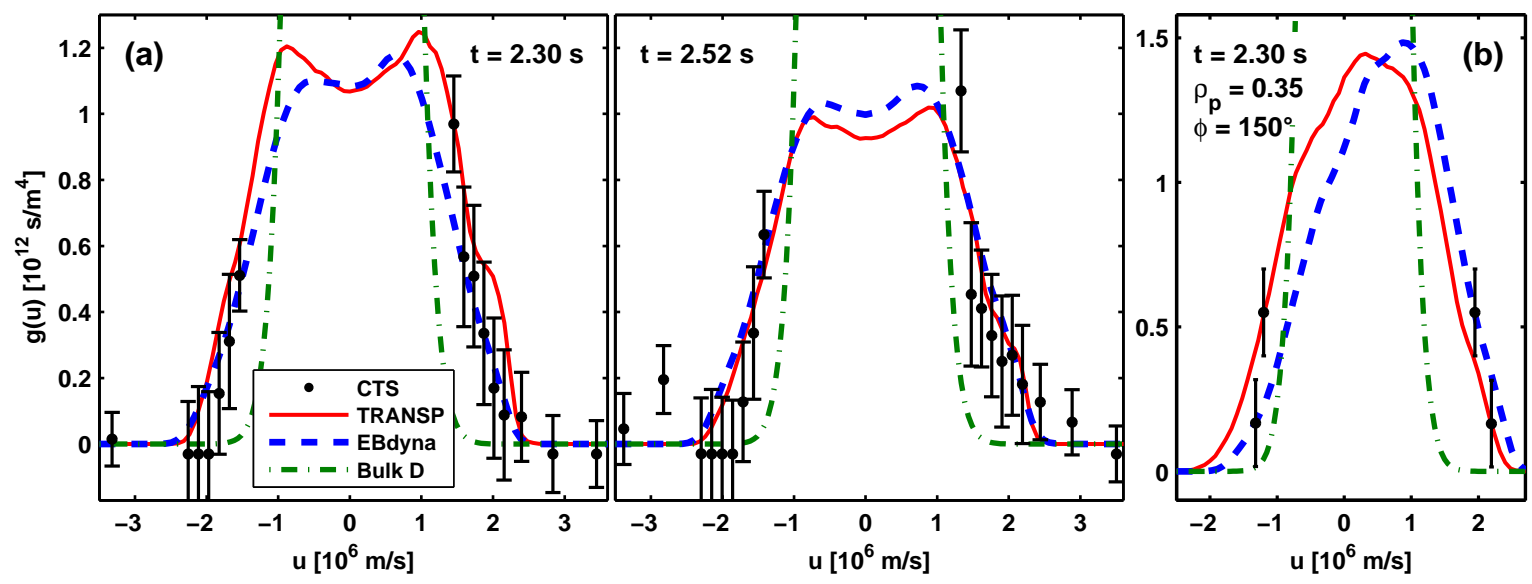

Figure 6. (a) 1D post-crash fast-ion distribution functions inferred from CTS compared to those predicted by TRANSP and EBdyna_go. The thermal-ion velocity distribution function (dash-dotted) is shown for reference. (b) Corresponding model predictions at $t=2.30 \mathrm{~s}$ for a different CTS location ( $\rho_{p}=0.35$ on the low-field side) and scattering geometry, with typical error bars from (a) overlayed for comparison.

Table 1. Sawtooth-induced fast-ion reduction in the CTS measurement volume at projected velocities $1.5 \times 10^{6} \mathrm{~m} / \mathrm{s}<|u|<3.5 \times 10^{6} \mathrm{~m} / \mathrm{s}$ for AUG \#30382.

\begin{tabular}{cccc}
\hline Crash & CTS & TRANSP & EBdyna_go \\
\hline$t=2.29 \mathrm{~s}$ & $40 \pm 24 \%$ & $44 \%$ & $59 \%$ \\
$t=2.51 \mathrm{~s}$ & $60 \pm 22 \%$ & $56 \%$ & $49 \%$ \\
\hline
\end{tabular}

here).

The post-crash 1D distribution functions in the CTS volume predicted by TRANSP and EBdyna_go are compared to the corresponding CTS results in figure 6 . When integrating over $1.5 \times 10^{6} \mathrm{~m} / \mathrm{s}<|u|<3.5 \times 10^{6} \mathrm{~m} / \mathrm{s}$ as done above, we confirm that EBdyna_go suggests a stronger fast-ion redistribution at $t=2.30 \mathrm{~s}$ than at $2.52 \mathrm{~s}(59$ and $49 \%$, respectively) in the observed velocity space, in contrast to the results from CTS and TRANSP. However, these redistribution levels remain consistent with those suggested by CTS within the uncertainties, implying that the overall redistribution inferred by CTS is in good agreement with both TRANSP and EBdyna_go. Table 1 summarizes the fast-ion reduction in the measurement volume across the two crashes as inferred from CTS, TRANSP, and EBdyna_go.

For attempts to discriminate between the two codes, the crash at $2.30 \mathrm{~s}$ is clearly the more useful from a CTS perspective, since the stronger redistribution predicted here by EBdyna_go is reflected in a lower $g(u)$ at nearly all $u$. In contrast, the two model predictions at $2.52 \mathrm{~s}$ differ only noticeably for projected velocities corresponding to those of the thermal bulk, where $g(u)$ remains unresolved by our measurements. At $2.30 \mathrm{~s}$, we note in particular the bump at $u \approx+2 \times 10^{6} \mathrm{~m} / \mathrm{s}$ seen in the TRANSP 
model only; comparison to figure $5(\mathrm{~d})$ shows that this derives from the full-energy NBI peak at $E=60 \mathrm{keV}$, which is much more prominent in TRANSP than in EBdyna_go at post-crash. However, this difference between the two codes is mainly an artefact of the different modelling approaches as discussed above. In light of this and of the experimental uncertainties, the present data do not clearly favour either of the two sawtooth models. Future CTS data of higher signal-to-noise ratio and with a scattering location and geometry optimized for the purpose should facilitate a more robust discrimination between the different predictions. As an example, figure 6(b) shows that a more toroidal projection angle further on the low-field side $\left(\phi=150^{\circ}\right.$, with a scattering location just within the sawtooth inversion radius at $\rho_{p}=0.4$ ) would yield larger differences in post-crash predictions at $t=2.30 \mathrm{~s}$. This is particularly true for negative projected velocities, where the model predictions are furthermore less affected by their differences in NBI treatment and averaging time.

In addition to sawtooth events, we highlight that fishbone instabilities also appear in this discharge at times close to some of the sawtooth crashes. These modes represent another manifestation of an $(m, n)=(1,1)$ perturbation in the plasma and can, under certain conditions, also give rise to significant anomalous fast-ion transport $[22,23]$. An example is presented in figure 7, showing the burst-like rise and slightly slower decay of magnetic perturbations characteristic of these modes. In contrast to the case of sawteeth, no clear, systematic impact of the fishbones on CTS spectra is observed, as illustrated in figure 7(b). Indeed, using the method above, we infer fast-ion redistribution levels for the times indicated in the figure which are consistent with zero. This represents the first CTS measurement of the impact of fishbones on fast ions at any device. Fishbones are driven by resonant interaction with trapped fast ions, and our results thus imply that these modes play a limited role in redistributing the passing fast ions expected to dominate the present CTS volume. There is also no evidence for fluctuations in the FILD signal correlated with these fishbone events. The conclusion that the observed fishbones do not significantly modify the inferred distribution function of core fast ions injected by the tangential NBI source Q3 extends recent results at AUG obtained from other diagnostics in different phase-space volumes [9].

\section{Discussion}

Our CTS results across two sawtooth crashes in AUG discharge 30382 indicate a 50\% reduction of fast ions in the measurement volume at $\rho_{p}=0.15$ in the observed velocity space, the first such CTS measurement at AUG. The result applies to passing fast ions, which strongly dominate the measurement volume in the scattering geometry considered here. We note that no systematic variations in neutron rates are observed across the two sawtooth crashes, suggesting that most of the sawtooth-redistributed fast ions remain confined. This is corroborated by the measurements from fast-ion loss detectors shown in figure 1(b), which indicate only a mild modulation in the FILD signal related to the sawtooth events, with typical increases in the signal of $10 \%$ following a crash. This is 

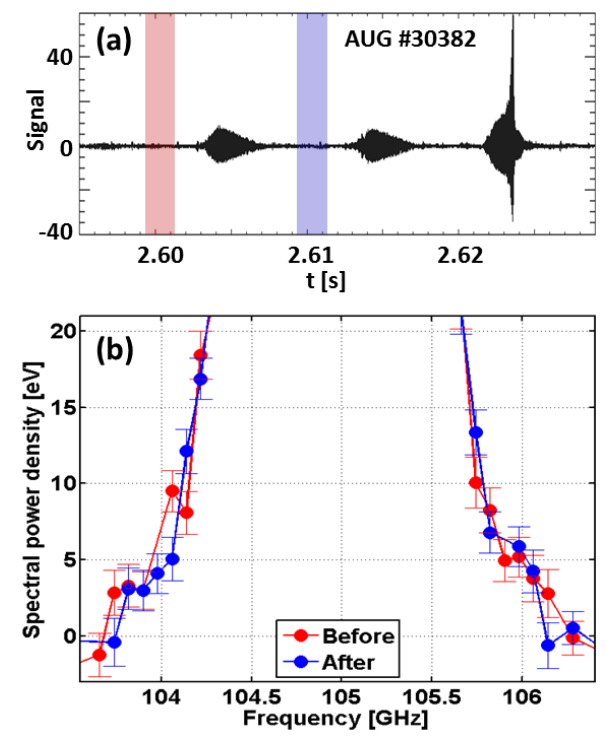

Figure 7. (a) Raw signal $(\mathrm{d} B / \mathrm{d} t$ in $[\mathrm{T} / \mathrm{s}])$ of Mirnov coil measurements around the time of the sawtooth crash at $t=2.62 \mathrm{~s}$. Shaded regions mark two consecutive CTS gyrotron-on periods just before and after a fishbone, for which the corresponding spectra are shown in (b).

in line with previous findings at AUG [7], confirming that sawteeth associated with fast ions generated by NBI source Q3 mostly redistribute fast ions rather than ejecting them from the plasma. In addition, the increase in the FILD signal after each sawtooth crash occurs on timescales of around $20 \mathrm{~ms}$, much longer than the actual crash duration from soft X-ray data of $\sim 0.1 \mathrm{~ms}$. This indicates that the increase is not due to losses induced directly by the crash but rather reflects the fact that fast ions are radially redistributed further off-axis by the sawteeth, from where they subsequently diffuse towards the edge.

The observed agreement between CTS measurements on the one hand and TRANSP and EBdyna_go on the other arises despite the fact that both codes assume full reconnection of the flux surfaces during a sawtooth crash, a condition which is often not satisfied $[24,25]$, including for about $40 \%$ of the crashes identified at AUG by automated methods [26]. For the two crashes discussed here, figure 8 indicates the presence of significant post-cursor activity at multiple frequencies, which might be related to incomplete reconnection. While this could suggest reduced fast-ion transport relative to the full-reconnection case, the consistency between the observed and simulated redistribution levels shows that both transport codes can nevertheless provide a satisfactory description of the CTS post-crash data under the conditions studied here. Further analysis of existing and forthcoming CTS data will improve our sawtooth statistics and allow us to assess the generality of this conclusion.

FIDA measurements in other sawtooth discharges similar to AUG\#30382 have recently been discussed in [8,9]; for AUG \#30815 (similar discharge parameters), these indicate a $30 \%$ fast-ion reduction on average, ranging up to $60 \%$ for strongly co-passing 


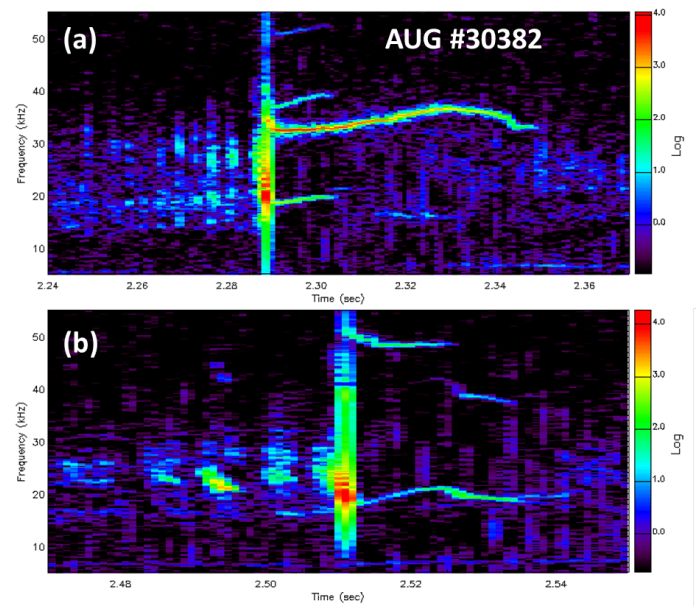

Figure 8. Mirnov coil magnetic spectrograms around the times corresponding to the sawtooth crashes at (a) $t=2.29 \mathrm{~s}$ and (b) $t=2.51 \mathrm{~s}$. Significant remnant MHD activity is seen at several frequencies following the sawtooth crashes.

ions, while results for AUG \#30809 (at slightly lower $B_{t}=-2.4 \mathrm{~T}$ ) suggest a central redistribution of about 50\%. Our results for AUG \#30382 itself are in line with these values. In this context, we note that the CTS geometry used here is sensitive to regions in fast-ion velocity space which overlap partially with those of FIDA at AUG [12], such that signals from the two diagnostics contain a contribution from the same subpopulation of fast ions. This has recently enabled a consistent combination of the FIDA and CTS fast-ion data for AUG \#30382 in a proof-of-principle analysis which considered the crash at $t=2.29 \mathrm{~s}$ also discussed here [27]. The results represent the first multidiagnostic tomographic reconstruction [28] of the 2D velocity distribution function across a sawtooth crash, and they corroborate those found here from CTS alone.

However, as illustrated by figure 5 and table 1, model predictions for the crashes at $t=2.29 \mathrm{~s}$ and $2.51 \mathrm{~s}$ differ somewhat, with EBdyna_go predicting a stronger overall redistribution than TRANSP at $t=2.29 \mathrm{~s}$ and a weaker one at $2.51 \mathrm{~s}$. Motivated by this difference, we use the techniques in [27] to present in figure 9 the corresponding combined FIDA and CTS tomographies for the crash at $t=2.51 \mathrm{~s}$, using a regularization based on minimum Fisher information (see [29] for details). The results confirm the clear sawtooth-induced reduction in central fast-ion density already inferred from CTS. Figure $9(\mathrm{c})$ shows the redistribution pattern in $(E, p)$ space, in the form of the relative change in the distribution function across the crash as implied by the tomographies. Co-passing ions with pitches around those of the NBI injection peaks are seen to suffer the strongest redistribution, while fast ions in the broad vicinity of zero pitch are also redistributed but significantly less so. Comparison to the CTS weight functions shown in figure 5 indicates that the 1D distribution function $g(u)$ inferred by CTS should be more strongly affected by the crash at positive projected velocities $u$ than at $u<0$. This is indeed observed, as discussed in section 3, thus highlighting the use of multi-diagnostic tomographies in interpreting measurements from individual fast-ion diagnostics. 

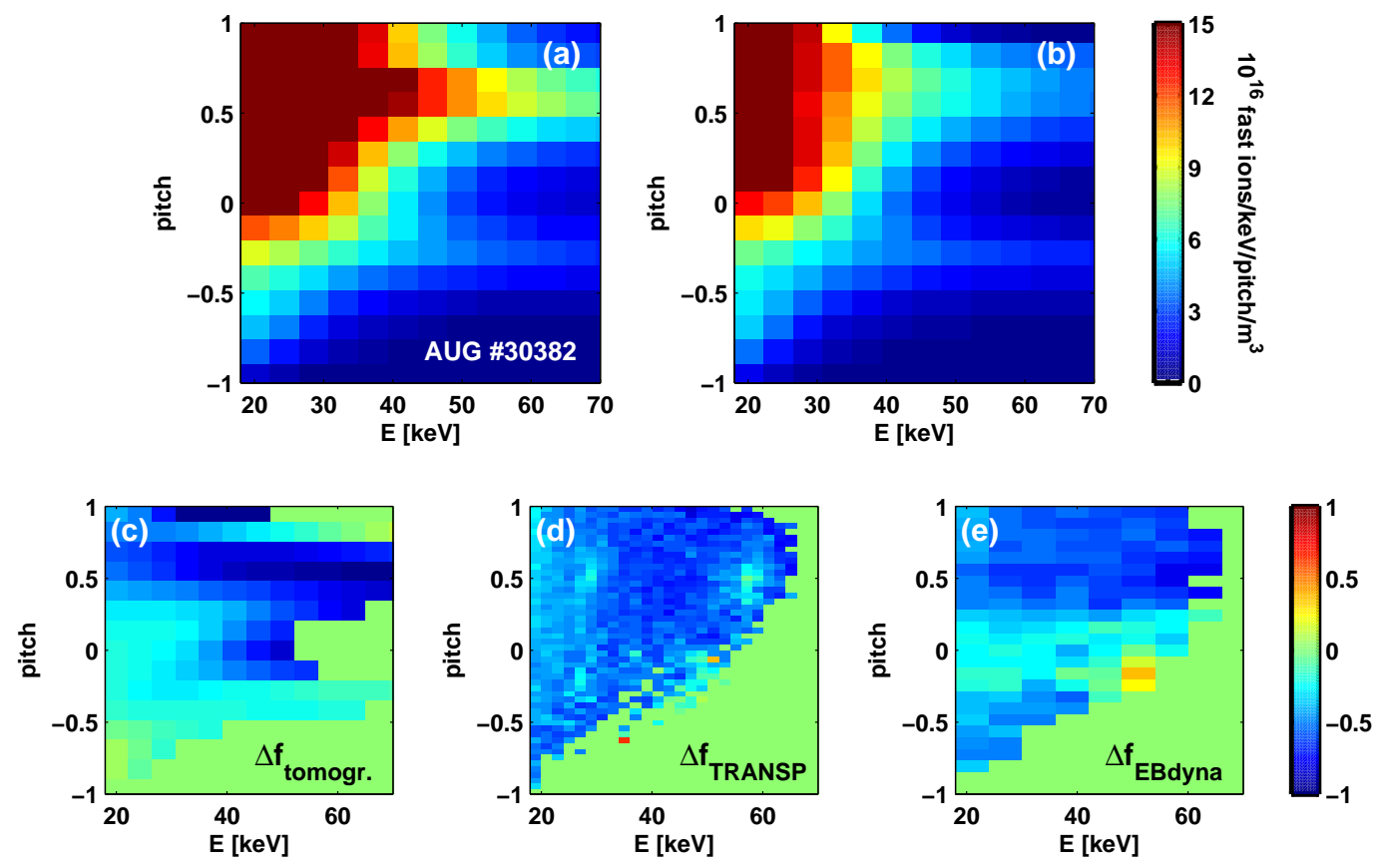

Figure 9. Velocity-space tomography of the fast-ion distribution function $f$ (a) before and (b) after the sawtooth crash at $t=2.51 \mathrm{~s}$, based on four FIDA views and one CTS view in AUG \#30382. Bottom panel shows the relative difference $\Delta f=$ $\left(f_{\text {after }}-f_{\text {before }}\right) / f_{\text {before }}$ from (c) the tomography and the corresponding predictions from (d) TRANSP and (e) EBdyna_go.

Further comparison of Figure 9(c) to the corresponding predictions from TRANSP and EBdyna_go in figure $9(\mathrm{~d})$ and (e) shows that the two codes agree on predicting a strong redistribution for co-passing ions, but the significantly weaker impact on ions around zero pitch suggested by the tomographies is better matched by EBdyna_go for this particular crash. When resampling the data in figures 9 (c)-(e) to the same resolution in $(E, p)$ space, we find a mean absolute difference between $\Delta f$ from the tomographies and from EBdyna_go of $0.17 \pm 0.02$, somewhat smaller than the corresponding value of $0.23 \pm 0.02$ for TRANSP. Despite this difference, the two codes give very similar predictions for the post-crash 1D distribution function $g(u)$ inferred from CTS in figure 6(a). This can be understood as a consequence of the broad shape of the CTS weight functions: Along the curves in $(E, p)$ space traced by these weight functions, the weak pitch dependence of the redistribution predicted by TRANSP is roughly balanced by the stronger redistribution at $|p|>0.5$ and weaker one at $|p|<0.5$ predicted by EBdyna_go.

As suggested by the above discussion, the results afforded by velocity-space tomography hold great promise for facilitating a more comprehensive view of the fastion transport seen in these and other experiments. In addition, acquisition of CTS sawtooth data at AUG with two simultaneous scattering geometries - analogous in this context to the multiple FIDA views - could not only enable useful 2D tomographies 
from CTS data alone, it could also enhance the ability of combined CTS and FIDA tomographies to discriminate between observed velocity-space redistribution patterns and those predicted by TRANSP and EBdyna_go. This can help to quantify to what extent transport mechanisms not included in TRANSP, such as $\mathbf{E} \times \mathbf{B}$ drifts, contribute to the sawtooth-driven fast-ion redistribution in certain regions of fast-ion velocityspace.

\section{Conclusions}

We have presented the first CTS measurements of sawtooth-induced redistribution of fast ions at AUG. Our results for two sawtooth crashes in AUG discharge 30382 indicate $\mathrm{a} \sim 50 \%$ reduction of fast ions in the measurement volume at $\rho_{p}=0.15$ in the observed velocity space. This also represents the first localized measurement of fast-ion redistribution on the high-field side in AUG. The result applies to passing fast ions and is in good agreement with predictions from the Kadomtsev model implemented in TRANSP, thus confirming previous results from FIDA measurements [8,9], and with the full-orbit EBdyna_go code. This represents the first demonstration of agreement between CTS sawtooth measurements and predictions of different transport codes. In addition, we find no evidence from CTS or FILD data that fishbone instabilities perturb the inferred fast-ion distribution function to a measurable degree. The present CTS measurements cannot clearly distinguish between the different model predictions for sawtooth-induced fast-ion redistribution, but the use of dedicated scattering geometries can enable a more robust discrimination between TRANSP and EBdyna_go based on CTS data alone. Finally, we use combined CTS and FIDA velocity-space tomography to map the fast-ion redistribution in energy-pitch space, showing that the result is slightly better matched by EBdyna_go than by TRANSP/Kadomtsev.

\section{Acknowledgements}

This work has been carried out within the framework of the EUROfusion Consortium and has received funding from the Euratom research and training programme 20142018 under grant agreement No 633053. The views and opinions expressed herein do not necessarily reflect those of the European Commission.

\section{References}

[1] Gorelenkov N.N. et al 2014 Nucl. Fusion 54125001

[2] von Goeler S. et al 1974 Phys. Rev. Lett. 331201

[3] Korsholm S.B. et al 2010 Nucl. Instrum. Methods Phys. Res. A 623677

[4] Salewski M. et al 2011 Nucl. Fusion 51083014

[5] Nielsen S.K. et al 2011 Nucl. Fusion 51063014

[6] Muscatello C.M. et al 2012 Plasma Phys. Control. Fusion 54025006

[7] Geiger B. et al 2014 Nucl. Fusion 54022005

[8] Geiger B. et al 2015 Nucl. Fusion 55083001 
[9] Geiger B. et al 2015 Plasma Phys. Control. Fusion 57014018

[10] Porcelli F. et al 1996 Plasma Phys. Control. Fusion 382163

[11] Nielsen S.K. et al 2015 Plasma Phys. Control. Fusion 57035009

[12] Rasmussen J. et al 2015 Plasma Phys. Control. Fusion 57075014

[13] Jaulmes F., Westerhof E. and de Blank H.J. 2014 Nucl. Fusion 54104013

[14] Garcia-Munoz M. et al 2009 Rev. Sci. Instrum 80053503

[15] Bindslev H. 1996 J. Atmos. Terr. Phys. 58 983-9

[16] Fischer R. et al 2010 Fusion Sci. Technol. 58 675-84

[17] Pankin A. et al 2004 Comp. Phys. Comm. 159 157-84

[18] Kolesnichenko Ya.I. and Yakovenko Yu.V. 1996 Nucl. Fusion 36159

[19] Salewski M. et al 2010 Nucl. Fusion 50035012

[20] Jaulmes F. et al 2016 This volume

[21] Heidbrink W.W. et al 2007 Plasma Phys. Control. Fusion 49 1457-1475

[22] Strachan J.D. et al 1985 Nucl. Fusion 25863

[23] Perez von Thun C. et al 2012 Nucl. Fusion 52094010

[24] Soltwisch H. 1992 Plasma Phys. Control. Fusion 34 1669-1698

[25] Beidler M.T. and Cassak P.A. 2011 Phys. Rev. Lett. 107255002

[26] van den Brand H. et al 2016 Nucl. Fusion submitted

[27] Jacobsen A.S. et al 2016 Plasma Phys. Control. Fusion submitted

[28] Salewski M. et al 2013 Nucl. Fusion 53063019

[29] Jacobsen A.S. et al 2016 Plasma Phys. Control. Fusion in press 